\title{
Meningococcal arthritis and myopericarditis: a case report
}

\author{
Lloyd Steele ${ }^{*}$, Katie Bechman, Eoghan De Barra and Charles Mackworth-Young
}

\begin{abstract}
Background: We report the first adult case of Neisseria meningitidis W-135 presenting with meningococcal arthritis and myopericarditis concomitantly, without other classical features of meningococcal disease.

Case presentation: A 67-year-old Caucasian man presented with acute-onset polyarthralgia, myalgia, and fever. On examination he had polyarticular synovitis. An electrocardiogram (ECG) demonstrated ST-elevation in leads I, II, III, aVF, and V2-V6 without reciprocal depression, and a high-sensitivity troponin level was significantly elevated. Cardiac magnetic resonance (CMR) imaging on day five of admission demonstrated patchy pericardial enhancement. Neisseria meningitidis W-135 was isolated from both synovial fluid and blood cultures. The clinical outcome was favourable with intravenous ceftriaxone and myopericarditis treatment (colchicine and ibuprofen).

Conclusions: We conclude that this is a rare case of disseminated Neisseria meningitidis W-135 presenting with acute polyarticular septic arthritis and myopericarditis, without other classical features of systemic meningococcal disease. The earlier described entity of primary meningococcal arthritis (PMA) can present in patients with meningococcal bacteraemia, and may not be distinct from disseminated meningococcal disease, but rather an atypical presentation of this.
\end{abstract}

Keywords: Neisseria meningitidis, Arthritis, Infectious, Meningococcal infections, Pericarditis

\section{Background}

Focal infections with meningococcus such as septic arthritis and pericarditis are often poorly recognised as presentations of meningococcal disease. We report the first adult case of Neisseria meningitidis $W-135$ presenting with meningococcal arthritis and myopericarditis concomitantly, without other classical features of meningococcal disease.

\section{Case presentation}

A 67-year-old Caucasian man presented with a one-day history of acute-onset polyarthralgia, myalgia, and fever. Apart from one episode of diarrhoea there were no other symptoms. He denied any precipitating trigger including recent sexual contact. He was born in South Africa but had lived in the UK for seven years prior to his presentation to our department. He had not travelled outside of the UK in that time. He was a non-smoker and worked as a glass collector in a kitchen. There was no history of intravenous drug use. His past medical history was significant for Ménière's disease.

\footnotetext{
* Correspondence: Iloyd.steele@imperial.nhs.uk; Lloyd.Steele@imperial.nhs.uk Charing Cross Hospital, Imperial College Healthcare NHS Trust, Fulham Palace Road, London W6 8RF, UK
}

(c) The Author(s). 2017 Open Access This article is distributed under the terms of the Creative Commons Attribution 4.0 International License (http://creativecommons.org/licenses/by/4.0/), which permits unrestricted use, distribution, and reproduction in any medium, provided you give appropriate credit to the original author(s) and the source, provide a link to the Creative Commons license, and indicate if changes were made. The Creative Commons Public Domain Dedication waiver (http://creativecommons.org/publicdomain/zero/1.0/) applies to the data made available in this article, unless otherwise stated.
His vital signs on admission showed a tachycardia (heart rate 101 beats per minute) and fever (temperature $38.0^{\circ} \mathrm{C}$ ), but were otherwise normal (respiratory rate $18 \mathrm{breaths} / \mathrm{min}$, SpO2 97\%, and blood pressure 107/79 mmHg). On examination he had polyarticular synovitis involving both knees (with moderate effusions), the right shoulder, left wrist, and right third proximal interphalangeal joint. There were no skin rashes and no peripheral lymphadenopathy. Cardiovascular examination was unremarkable.

Laboratory investigations revealed lymphopaenia $(0.2 \times$ $10^{9}$ cells/L), thrombocytopaenia $\left(128 \times 10^{9}\right.$ cells/L), anaemia (haemoglobin $105 \mathrm{~g} / \mathrm{L}$ ), an acute kidney injury (creatinine $129 \mu \mathrm{mol}$ (89\% increase from baseline)), and an acute phase response (C-reactive protein (CRP) $309 \mathrm{mg} / \mathrm{L}$ and erythrocyte sedimentation rate (ESR) $39 \mathrm{~mm} / \mathrm{h}$ ). An autoimmune screen (rheumatoid factor, antinuclear antibody, and anti-neutrophil cytoplasmic antibody) was negative. Complement 3, complement 4, $\mathrm{CH} 50$ level, and alternate pathway complement function were normal. Urine microscopy revealed only scanty red cells and $<50$ white cells per cubic millimetre.

An electrocardiogram (ECG) demonstrated ST-elevation in leads I, II, III, aVF, and V2-V6 without reciprocal 
depression (Fig. 1). A high-sensitivity troponin I level was significantly elevated at 33,169 $\mathrm{ng} / \mathrm{L}(0-34 \mathrm{ng} / \mathrm{L})$ with a peak of $48,140 \mathrm{ng} / \mathrm{L}$ at $12 \mathrm{~h}$ post-admission. An urgent echocardiogram using a pocket-sized ultrasound device (V-scan) on day one demonstrated mild-moderate left ventricular impairment with no regional wall motion abnormalities. Cardiac magnetic resonance (CMR) imaging on day five of admission demonstrated patchy pericardial enhancement with no obvious myocardial enhancement (Fig. 2).

Aspiration from the right knee yielded a purulent fluid, and microscopy revealed Gram negative cocci, monocytes, and polymorphs. Neisseria meningitidis W-135 was isolated from both synovial fluid and blood cultures. The serotype was confirmed at Public Health England's Meningococcal Reference Unit using standard methods [1] (see Additional file 1).

The patient was commenced on treatment for myopericarditis with colchicine and ibuprofen after Cardiology assessment. Intravenous ceftriaxone $2 \mathrm{~g}$ twice per day was commenced after review by the Infectious Diseases team, initially due to a suspected diagnosis of disseminated gonococcal infection. Azithromycin treatment was not given due to reports of causing colchicine toxicity. The patient did not develop nuchal rigidity or other signs of meningism or meningococcaemia. His fever resolved by day three with a subsequent improvement in the inflammatory response. Troponin levels were monitored daily and fell sharply, normalising after 5 days of therapy, and a departmental echocardiogram at this point demonstrated improved ventricular function. After two weeks of treatment the lymphopaenia and thrombocytopaenia had resolved, and renal function had returned to baseline. Intravenous ceftriaxone was then switched to oral ciprofloxacin $500 \mathrm{mg}$ twice per day which was continued for a further week.

His musculoskeletal symptoms were slower to improve despite regular non-steroidal anti-inflammatory medication

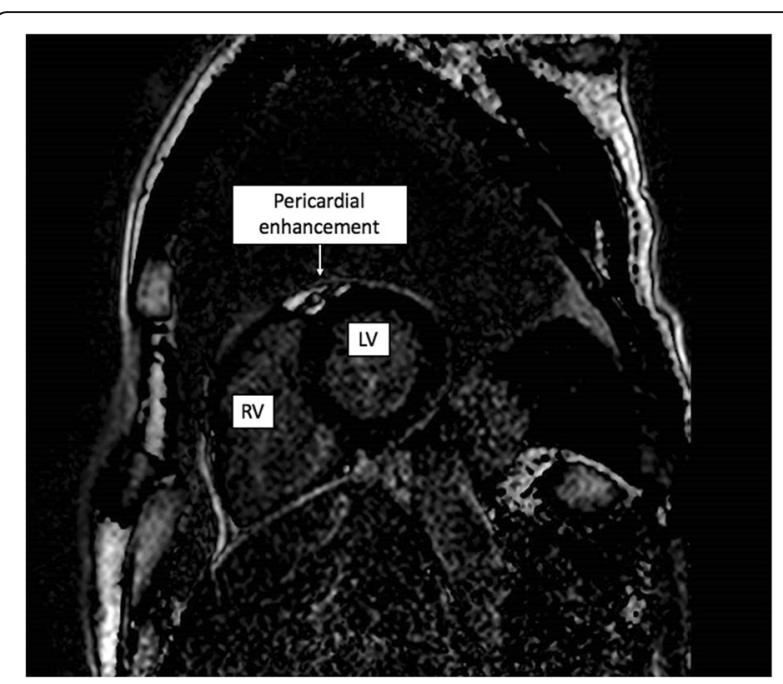

Fig. 2 Cardiac magnetic resonance (CMR) short-axis stack image showing the left (LV) and right ventricles (RV), with pericardial enhancement

and physiotherapy. Synovitis at the shoulder and wrist persisted for approximately 6 weeks. He developed symptoms of carpal tunnel syndrome on day 6 , which had not resolved by outpatient review at 8 weeks. At clinic review he stated his intention to return to South Africa on a permanent basis. He was advised to consider carpal tunnel release in South Africa if his symptoms continued.

\section{Discussion}

Acquisition of meningococcus is usually through very close contact with respiratory secretions or saliva $[2,3]$. It can asymptomatically colonise the nasopharynx, as in $8-25 \%$ of healthy individuals $[2,4]$, or cause invasive disease [2]. The mechanisms that lead from colonisation to invasive disease are still incompletely understood, but are thought to be a result of environmental conditions, host susceptibility, and meningococcal virulence factors

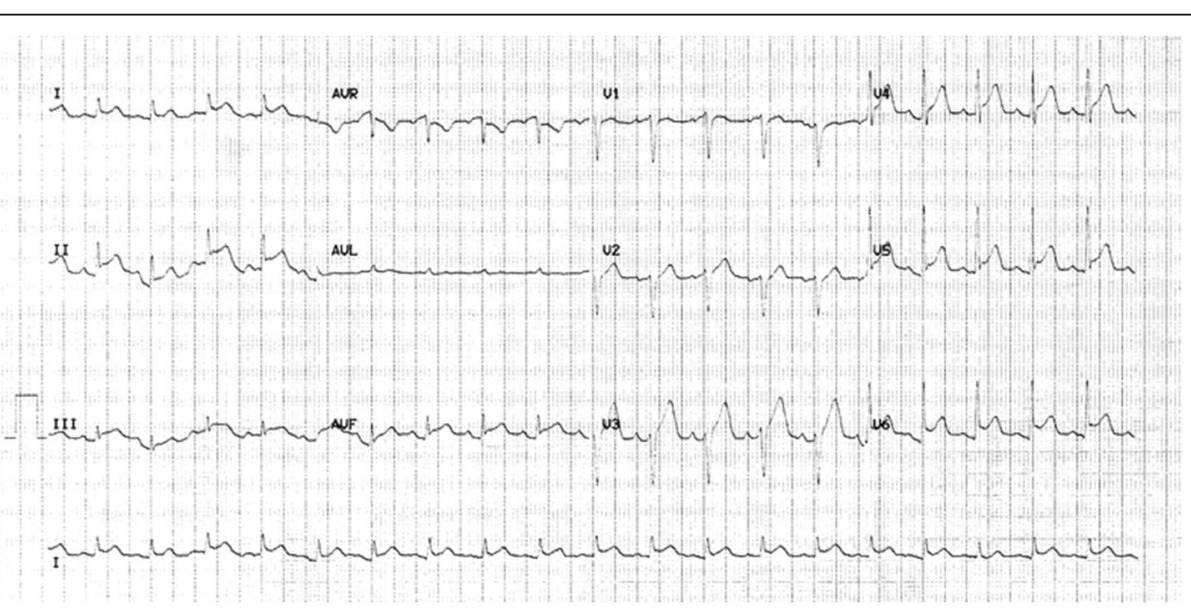

Fig. 1 Twelve lead electrocardiogram showing ST-elevation in leads I, II, III, aVF, and V2-V6 without reciprocal depression 
$[2,5]$. The degree of virulence of $N$. meningitidis is influenced by capsule expression, which itself is used to define the individual serogroups [2]. Although 13 distinct serogroups exist, the majority of invasive meningococcal diseases are caused by only six of these: A, B, C, W, X, and $Y[2,3,5]$. In Europe, serogroups $B$ and $C$ cause the majority of invasive disease cases [2]. Since 2009, the number of serogroup W cases in England has been increase year-on-year, from 19 in 2008/2009 to 176 in 2014/2015 (a quarter of all laboratory-confirmed meningococcal cases that year), with a rise in the hypervirulent ST-11 stain [6]. Interestingly, during the twenty-first century, serogroup $\mathrm{W}$ has become the predominant cause of invasive disease in all age groups in South Africa, in place of serogroup A $[7,8]$. However, the overall incidence of meningococcal disease in South Africa is at an all-time low, with an average incidence in the population of 1 case per 100,000 population [9].

The two most common clinical presentations of invasive meningococcal disease are meningitis and the often fatal syndrome of acute meningococcaemia [5]. The ability of $N$. meningitidis to cause disease beyond these presentations is often overlooked [10]. Other presentations include pericarditis, conjunctivitis, panophthalmitis, pneumonia, urogenital tract infections, and arthritis [2].

Arthritic involvement is not an uncommon feature of invasive meningococcal disease, occurring in up to $11 \%$ of adult cases [11]. The majority of this occurs in patients with acute meningococcaemia with direct haematogenous seeding of circulating bacteria $[11,12]$. Two other clinical patterns of meningococcal arthritis are also described [11, 12]: an immune-mediated arthritis, characterised by sterile purulent effusions, and an acute septic arthritis in patients without meningitis or the classical syndrome of meningococcaemia, defined as the combination fever, rash, and hemodynamic instability [11]. Earlier studies have defined this as primary meningococcal arthritis (PMA) [11, 12]. However the classification of PMA is somewhat confusing. The synovium is positive for meningococcus in $90 \%$ of cases [12], but $N$. meningitidis is also cultured from the blood in $40 \%$ of cases [13]. Additionally the infection is likely to originate outside of the joint, and thus characterising cases with bacteraemia as PMA may be problematic and a general description of meningococcal septic arthritis may be more appropriate.

PMA remains a rare diagnosis, with only 46 cases reported up to 2016 [14]. Presentation of PMA may be very similar to that of other forms of septic arthritis [12]. It is most commonly monoarticular [15], with the knee the most commonly involved joint [12, 14, 16], but can also be oligoarticular or polyarticular [12, 16]. Causative serotypes are group B, 30-38\%; group C, 33-36\%; and group W-135, 13-29\% [15, 17]. The majority of cases due to group W-135 are in children $[15,18-21]$, with only one case reported in an adult [13]. Our patient did not have meningism or the features of acute meningococcaemia, and thus his condition fits with a diagnosis of PMA.

In addition to joint involvement, our patient presented with myopericarditis. Meningococcal pericarditis is an unusual but well-described complication of meningococcus, occurring in 3-19\% of cases [22-24]. As with articular involvement, it can be classified into three categories: a process secondary to disseminated disease (purulent; culture-positive; associated with widespread, clinically-manifested meningococcal disease); a reactive immune-mediated process (immunological, late-onset, culture-negative); or a primary infection (purulent, culturepositive, but without evidence of meningeal involvement or clinical manifestations of meningococcaemia) [25]. Common features include ECG abnormalities (93\%), chest pain (84\%), and fever (69\%) [22]. Cardiac tamponade is also a recognised complication, and in such cases pericardiocentesis, a pericardial window, or even a pericaridiotomy may be required [25]. Features which can suggest concomitant myocardial involvement (myocarditis) include dyspnoea, palpitations, fatigue, and a troponin rise [26], with the latter being a prominent part of our patient's presentation.

The prognosis for arthritic involvement in meningococcal arthritis is excellent. In one study although 33\% of patients were treated with steroids for up to several months due to persisting symptoms, all patients recovered and there was no joint deformity or impairment at longterm follow-up [27]. The literature suggests that recovery from acute meningococcal myocarditis can occur within $3-6$ days $[28,29]$, a similar time course to that observed in our case.

Preventing invasive meningococcal disease has been an issue of great importance in public health. An effective conjugate group $\mathrm{C}$ vaccine was introduced in the $\mathrm{UK}$ in 1999, and then conjugate group A vaccines in the African meningitis belt beginning in 2010 [30], but throughout this time developing a vaccine against serogroup B had been a challenge. However, recently, there have been reports of early efficacy for the serogroup $B$ vaccine in the UK [31], which now has a comprehensive programme against serogroups A, B, C, W, and Y. Ongoing surveillance will be needed to assess the longer-term impact of this.

\section{Conclusions}

We report a rare case of disseminated Neisseria meningitidis W-135 presenting with acute polyarticular septic arthritis and myopericarditis, without other classical features of systemic meningococcal disease. The earlier described entity of PMA can present in patients with meningococcal bacteraemia, and may not be distinct from disseminated meningococcal disease, but rather an atypical 
presentation of this. Meningococcus should be included in the differential diagnosis of focal infections including septic arthritis and myopericarditis in the absence of meningitis or meningococcaemia.

\section{Additional file}

Additional file 1: Public Health England Meningococcal Reference Unit User Manual. (PDF 816 kb)

\section{Abbreviations}

CMR: Cardiac Magnetic Resonance; CRP: C-Reactive Protein; ECG: Electrocardiogram; ESR: Erythrocyte Sedimentation Rate; PMA: Primary Meningococcal Arthritis

\section{Acknowledgements}

We would like to acknowledge Dr. Steve Gray for providing information from the Meningococcal Reference Unit.

\section{Funding}

No funding

\section{Availability of data and materials}

Data sharing is not applicable to this article as no datasets were generated or analysed during the current study.

\section{Authors' contributions}

$L S, K B, E D B$, and $C M Y$ were involved in the management of the clinical case during the patient's admission. LS and KB drafted the manuscript and EDB and CMY provided critical review. All authors read and approved the final manuscript.

\section{Ethics approval and consent to participate}

Not applicable

\section{Consent for publication}

Patient consent for case report publication in journal and has signed consent form

\section{Competing interests}

The authors declare that they have no competing interests.

\section{Publisher's Note}

Springer Nature remains neutral with regard to jurisdictional claims in published maps and institutional affiliations.

Received: 22 July 2017 Accepted: 21 November 2017

Published online: 06 December 2017

\section{References}

1. Public Health England. Meningococcal reference unit user manual. Manchester: Public Health England; 2015.

2. Stephens DS, Greenwood B, Brandtzaeg P. Epidemic meningitis, meningococcaemia, and Neisseria meningitidis. Lancet. 2007;369(9580): 2196-210

3. Rouphael NG, Stephens DS. Neisseria meningitidis: biology, microbiology, and epidemiology. Methods Mol Biol. 2012;799:1-20.

4. Cartwright KA, Stuart JM, Jones DM, Noah ND. The Stonehouse survey: nasopharyngeal carriage of meningococci and Neisseria lactamica. Epidemiol Infect. 1987;99(3):591-601.

5. Pace D, Pollard AJ. Meningococcal disease: clinical presentation and sequelae. Vaccine. 2012;30(Suppl 2):B3-9.

6. Ladhani SN, Ramsay M, Borrow R, Riordan A, Watson JM, Pollard AJ. Enter B and W: two new meningococcal vaccine programmes launched. Arch Dis Child. 2016:101(1):91-5.

7. Zuckerman JN, Broker M, Worth C. 2010 FIFA world cup South Africa: travel health issues and new options for protection against meningococcal disease. Travel Med Infect Dis. 2010;8(2):68-73.
8. von Gottberg A, du Plessis M, Cohen C, Prentice E, Schrag S, de Gouveia L, et al. Emergence of endemic serogroup W135 meningococcal disease associated with a high mortality rate in South Africa. Clin Infect Dis. 2008;46(3):377-86.

9. Meiring S, Hussey G, Jeena P, Parker S, von Gottberg S. Recommendations for the use of meningococcal vaccines in South Africa. South Afr J Infect Dis. 2017:32(3):82-6.

10. Dillon M, Nourse C, Dowling F, Deasy P, Butler K. Primary meningococcal arthritis. Pediatr Infect Dis J. 1997;16(3):331-2.

11. Schaad UB. Arthritis in disease due to Neisseria meningitidis. Rev Infect Dis. 1980;2(6):880-8.

12. Harwood MI, Womack J, Kapur R. Primary meningococcal arthritis. J Am Board Fam Med. 2008;21(1):66-9.

13. Moll-Manzur C, Faundez F, Araos-Baeriswyl E, Monsalve X. [Septic arthritis of the knee by Neisseria meningitidis serogroup W-135: First case reported in adults]. Med Clin (Barc). 2016;147(5):225-6.

14. Straticiuc S, Ignat A, Hanganu E, Lupu W, Ciubara AB, Cretu R. Neisseria meningitidis Serogroup $C$ causing primary arthritis in a child: case report. Medicine (Baltimore). 2016;95(5):e2745.

15. Vienne P, Ducos-Galand M, Guiyoule A, Pires R, Giorgini D, Taha MK, et al. The role of particular strains of Neisseria meningitidis in meningococcal arthritis, pericarditis, and pneumonia. Clin Infect Dis. 2003;37(12):1639-42.

16. Sarinho JC, Arcadipane MS, Menezes GT, Duarte DF, Cossermelli W, Aprahamian I. Primary meningococcal polyarthritis in an adult woman. Case Rep Med. 2015:2015:563672.

17. Efrati O, Barak A, Yahav J, Leibowitz L, Keller N, Bujanover Y. Primary meningococcal arthritis. Isr Med Assoc J. 2002:4(5):386-7.

18. Apfalter $\mathrm{P}$, Horler R, Nehrer S. Neisseria meningitidis serogroup W-135 primary monarthritis of the hip in an immunocompetent child. Eur J Clin Microbiol Infect Dis. 2000;19(6):475-6.

19. McMullan B. An infant with meningococcal arthritis of the hip. J Paediatr Child Health. 2009:45(12):762-3.

20. Brawley RL, Barson WJ, Palmer R, Hilty MD, Koranyi K. Acute septic arthritis caused by Neisseria meningitidis serogroup W-135. South Med J. 1980;73(3): 395-6.

21. Faye A, Mariani-Kurkdjian P, Taha MK, Angoulvant F, Antonios M, Aubertin G, et al. Clinical features and outcome of pediatric Neisseria meningitidis serogroup W135 infection: a report of 5 cases. Clin Infect Dis. 2004:38(11):1635-7.

22. Blaser MJ, Reingold AL, Alsever RN, Hightower A. Primary meningococcal pericarditis: a disease of adults associated with serogroup C Neisseria meningitidis. Rev Infect Dis. 1984;6(5):625-32.

23. Morse JR, Oretsky MI, Hudson JA. Pericarditis as a complication of meningococcal meningitis. Ann Intern Med. 1971:74(2):212-7.

24. Herrick W. Meningococcal pericarditis, with report of 12 cases. Med Clin North Am. 1918:2:411-26.

25. Finkelstein $Y$, Adler $Y$, Nussinovitch $M$, Varsano I, Amir J. A new classification for pericarditis associated with meningococcal infection. Eur J Pediatr. 1997: 156(8):585-8.

26. Thanjan MT, Ramaswamy P, Lai WW, Lytrivi ID. Acute myopericarditis after multiple vaccinations in an adolescent: case report and review of the literature. Pediatrics. 2007:119(6):e1400-3.

27. Cabellos C, Nolla JM, Verdaguer R, Pelegrin I, Ribera A, Ariza J, et al. Arthritis related to systemic meningococcal disease: 34 years' experience. Eur J Clin Microbiol Infect Dis. 2012;31(10):2661-6.

28. Razminia M, Salem Y, Elbzour M, Teves D, Deshmukh H, Khosla S. Importance of early diagnosis and therapy of acute meningococcal myocarditis: a case report with review of literature. Am J Ther. 2005;12(3):269-71.

29. Shrestha P, Shrestha NK, Giri S. Rapid recovery following fulminant meningococcemia complicated by myocarditis in a 15-year-old Nepalese girl: a case report. Int Med Case Rep J. 2013;6:33-6.

30. Basta NE, Christensen H. 4CMenB vaccine effectiveness: reasons for optimism. Lancet. 2016:388(10061):2719-21.

31. Parikh SR, Andrews NJ, Beebeejaun K, Campbell H, Ribeiro S, Ward C, et al. Effectiveness and impact of a reduced infant schedule of $4 \mathrm{CMen} B$ vaccine against group B meningococcal disease in England: a national observational cohort study. Lancet. 2016;388(10061):2775-82. 